\title{
Crecimiento de juveniles de la corvina aguada, Cynoscion squamipinnis (Perciformes: Sciaenidae) en cautiverio
}

\author{
Growth of Weakfish Hatchery-produced Juveniles, Cynoscion Squamipinnis \\ (Perciformes: Sciaenidae) in Captivity
}

\author{
Jorge Boza-Abarca \\ jorge.boza.abarca@una.cr \\ Estación de Biología Marina Juan Bertoglia Richards \\ Escuela de Ciencias Biológicas \\ Universidad Nacional \\ Puntarenas, Costa Rica \\ Marvin Ramírez-Alvarado \\ marvin.ramírez.alvarado@una.cr \\ Estación de Biología Marina Juan Bertoglia Richards \\ Escuela de Ciencias Biológicas \\ Universidad Nacional \\ Puntarenas, Costa Rica \\ Juan Barquero-Chanto \\ juanes0489@gmail.com \\ Estación de Biología Marina Juan Bertoglia Richards \\ Escuela de Ciencias Biológicas \\ Universidad Nacional \\ Puntarenas, Costa Rica \\ Emilia Calvo-Vargas \\ emilia.calvo.vargas@una.cr \\ Estación de Biología Marina Juan Bertoglia Richards \\ Escuela de Ciencias Biológicas \\ Universidad Nacional \\ Puntarenas, Costa Rica \\ Karen Berrocal-Artavia \\ karen.berrocal.artavia@una.cr \\ Estación de Biología Marina Juan Bertoglia Richards \\ Escuela de Ciencias Biológicas \\ Universidad Nacional \\ Puntarenas, Costa Rica
}

Recibido-Received: 27/ago/2015 / Aceptado-Accepted: 15/nov/2015 / Publicado-Published: 31/jul/2016. 
UNICIENCIA Vol. 30, No. 2, pp. 63-74. Julio-diciembre, 2016.

ISSN Electrónico: 2215-3470

URL: www.revistas.una.ac.cr/uniciencia

DOI: http://dx.doi.org/10.15359/ru.30-2.5

Email: revistauniciencia@una.cr

\title{
Resumen
}

Peces juveniles de corvina aguada, Cynoscion squamipinnis, obtenidos a partir de desoves espontáneos en cautiverio fueron engordados por 355 días en la Estación de Biología Marina Juan Bertoglia Richards (Costa Rica). Ciento cincuenta y dos peces fueron sembrados en tanques de cuatro toneladas a una biomasa total de $82 \mathrm{~g} \mathrm{~m}^{-3}$, y alimentados con una dieta de $50 \%$ proteína, $17.5 \%$ carbohidratos, $11.8 \%$ lípidos, $16 \%$ ceniza y $5 \%$ humedad. El oxígeno disuelto fue de $7.12 \pm 1.13 \mathrm{mg} \mathrm{L}^{-1}$ (5.68 - 9.13 $\left.\mathrm{mg} \mathrm{L}^{-1}\right)$, la salinidad de $31.92 \pm 1.65$ ups (29.84 - 34.71 ups), y la temperatura de $27.54 \pm 0.63^{\circ} \mathrm{C}(26.50$ $28.60^{\circ} \mathrm{C}$ ), condiciones similares a las del Golfo de Nicoya. El peso inicial promedio fue de $3.77 \pm 1.15 \mathrm{~g}$ (0.3 - 6.2 g). El peso promedio final fue de $132.60 \pm 32.96 \mathrm{~g}(78.5-219.3 \mathrm{~g})$. El crecimiento (C) fue de $128.83 \mathrm{~g}$, con una tasa de crecimiento (TC) de $0.36 \mathrm{~g} \mathrm{~d}^{-1}$, y una tasa específica de crecimiento (TEC) de $1.00 \% \mathrm{PC} \mathrm{d}^{-1}$. La tasa de crecimiento relativa (TCR) fue de $0.0053 \mathrm{~g} \mathrm{~g}^{-1} \mathrm{~d}^{-1}$. El factor de conversión alimenticia (FCA) durante todo el periodo fue de $1.8 \pm 0.4$. La corvina aguada tuvo un crecimiento menor al de otras corvinas, mostró un comportamiento tranquilo, aceptó la dieta peletizada y fue fácil de manipular, por lo que es candidata para la maricultura.

Palabras claves: Biología marina, acuicultura, crecimiento; pez marino, corvina aguada, Cynoscion squamipinnis.

\begin{abstract}
Juveniles of weakfish, Cynoscion squamipinnis hatchery-produced from spontaneously spawning in captivity, were grown during 355 days at Estación de Biología Marina Juan Bertoglia Richards (Biology Marine Station John Bertoglia Richards) in Costa Rica. One hundred fifty two fish were stocked in tanks of four tonnes to a total biomass of $82 \mathrm{~g} \mathrm{~m}-3$, and feeding with a diet of $50 \%$ protein, $17.5 \%$ carbohydrate, $11.8 \%$ lipids, $16 \%$ ash and $5 \%$ humidity. Dissolved oxygen was $7.12 \pm 1.13 \mathrm{mg} \mathrm{L}-1$ (5.68 - 9.13 mg L- 1), salinity of $31.92 \pm 1.65$ ups (29.84 - $34.71 \mathrm{ups})$, and temperature of $27.54 \pm 0.63^{\circ} \mathrm{C}$ $\left(26.50-28.60^{\circ} \mathrm{C}\right)$, similar to the Gulf of Nicoya conditions. Initial mean weight was $3.77(1.15 \mathrm{~g}(0.3$ $6.2 \mathrm{~g})$. Final mean body weight was 132.60 (32.96 g (78.5 - 219.3 g). The growth (G) was $128.83 \mathrm{~g}$, with a growth rate (GR) of $0.36 \mathrm{~g} \mathrm{~d}-1$, and Specific Growth Rate (SGR) of $1.00 \% \mathrm{BW} \mathrm{d}-1$. The Relative Growth Rate (RGR) was $0.0053 \mathrm{~g}$ g- $1 \mathrm{~d}-1$. The feed conversion (FCA) during the period was $1.8 \pm 0.4$. The weakfish had a lower growth parameters than other croaker species, the fish showed a calm behavior, accepted the pelletized diet, and they were easy to handle, characteristics which confirm that weakfish as potential candidate for commercial mariculture.
\end{abstract}

Keywords: Marine biology, aquaculture, growth; sea fish, weakfish, Cynoscion squamipinnis.

Las corvinas pertenecen a la familia Sciaenidae, se les conoce como roncadores debido a los sonidos que producen durante el cortejo de reproducción (Connaughton y Taylor, 1996; Lagárdere y Marianis, 2006), y se distribuyen en las regiones templadas y tropicales de todo el mundo (ذiménez et al., 2005).

En un esfuerzo para diversificar la producción en acuicultura, la investigación se ha centrado en la búsqueda de nuevas especies con un futuro prometedor (Chatzifotis et al., 2006). Los esciánidos se han cultivado con diferentes propósitos; destaca el corvinón ocelado (Sciaenops ocellatus), criado para repoblar áreas naturales o como alimento en los Estados Unidos; la especie Totoaba macdonaldi en el Golfo de California para su repoblación (López et al., 2006) y la cobia (Rachycentron canadum) en el Golfo de México y la costa del Mar Caribe (Benetti et al., 2007) para su cultivo; la corvina japonesa (Argyrosomus 
ISSN Electrónico: 2215-3470 DOI: http://dx.doi.org/10.15359/ru.30-2.5
UNICIENCIA Vol. 30, No. 2, pp. 63-74. Julio-diciembre, 2016. URL: www.revistas.una.ac.cr/uniciencia Email: revistauniciencia@una.cr

japonicus) en Sur África (Bernatzeder et al., 2010) y en Australia; el verrugato (Umbrina cirrosa) en el Mediterráneo (ذiménez et al., 2005) y, recientemente, la corvina mediterránea (Argyrosomus regius) en España (Cárdenas, 2011; Cárdenas, 2012; Duncan et al., 2012) y en Grecia (Chatzifotiz et al., 2010; Mylonas et al., 2013a; Mylonas et al., 2013b), así como en Francia, Italia y Marruecos (ذiménez et al.,2005; Monfort, 2010; Piccolo, 2018) para su cultivo comercial.

La corvina aguada Cynoscion squamipinnis (Sciaenidae) es una de las 34 especies distribuidas a lo largo de la costa pacífica de América, y en Costa Rica es de importancia comercial junto con la corvina reina, C. albus (ذiménez et al., 2005; Vásquez y Marín, 2012). Sin embargo, su captura ha disminuido debido a la presión pesquera (Chacón et al., 2007; Marín y Vásquez, 2012), de ahí el interés en realizar estudios sobre su reproducción y crecimiento en cautiverio, con el fin de implementar proyectos de maricultura, similar a lo que ocurre con cerca de 18 especies que se crían en el mundo (Cárdenas, 2011; 2012; Duncan et al., 2012).

El crecimiento de estos peces durante la fase de preengorde (2 - 3 meses) y engorde (hasta 24 meses) ha sido comparado para diferentes especies y en diferentes circunstancias. En general, los esciánidos crecen hasta los 50 g en $2-3$ meses con tasas específicas de crecimiento diario de 3\% peso corporal (PC) $\mathrm{d}^{-1}$, con supervivencias entre $60-100 \%$. A. japonicus tardó 16 meses para llegar a los $800 \mathrm{~g}$ en estanques (talla comercial), y alrededor de 24 meses hasta los $1000 \mathrm{~g}$ en tanques. S. ocellatus cultivado en estanques durante 18 meses, alcanzó un peso de $1178 \mathrm{~g}$, y cultivado en viveros flotantes durante 12 meses, llegaron a pesar $798 \mathrm{~g}$. Por último, C. gilberti creció $480 \mathrm{~g}$, pero los intervalos de tiempo para alcanzar ese peso variaron de 8 a 13 meses (Cárdenas, 2011).

La cobia ( $R$. canadum) es la especie más prometedora, con tasas de crecimiento superiores a cualquier otra especie de corvina, y cuya tecnología ha sido mejorada en Taiwán, para ponerla a disposición en los países de América, específicamente en el Caribe, con crecimientos de $1.5 \mathrm{~g}$ hasta $6 \mathrm{Kg}$ en 12 meses (Benetti et al., 2007).

Con respecto a T. macdonaldi, López et al. (2006) reportaron un crecimiento de $200.1 \pm 82.5 \mathrm{~g}$ después de 1 año de cultivo, las cuales fueron alimentadas con una dieta alta en proteína $(66.9 \pm 1.4 \%)$.

En Costa Rica, los estudios sobre la reproducción y crecimiento de especies marinas iniciaron con el pargo mancha, Lutjanus guttatus (Boza-Abarca et al., 2008, 2011), generando la experiencia para lograr la reproducción espontánea en cautiverio de la corvina aguada $(C$. squamipinnis), la descripción de su desarrollo ontogénico, y la comparación de su crecimiento con el de otras especies (en revisión). El objetivo de este estudio fue evaluar el crecimiento en cautiverio de los juveniles producidos en estos desoves espontáneos, con el fin de contribuir al conocimiento acerca de esta especie de interés comercial.

\section{Metodología}

Los juveniles de corvina aguada (C. squamipinnis) utilizados en este experimento, fueron la primera generación obtenida en cautiverio en la Estación de Biología Marina Juan Bertoglia Richards de la Universidad Nacional en Puntarenas, a partir de un desove espontáneo de peces silvestres colectados en el Golfo de Nicoya, Costa Rica. Los peces fueron criados hasta 
completar el periodo larval e iniciar el periodo juvenil (45 días después del desove), y luego fueron distribuidos en dos tanques de engorde de $4 \mathrm{~m}^{3}$ (3.5 $\mathrm{m}^{3}$ de agua total), con una biomasa total de $82 \mathrm{~g} \mathrm{~m}^{3}$ por tanque, aireación constante (soplador eléctrico), recambio de agua (100\% diario) y alimentador automático de banda (12 h).

Para todo el periodo de crecimiento, se midieron los parámetros ambientales cada día, durante la mañana. La salinidad se midió con un refractómetro (ups), y el oxígeno disuelto (mg $\left.\mathrm{L}^{-1}\right)$ y la temperatura $\left({ }^{\circ} \mathrm{C}\right)$ con un multiparámetros YSI EcoSense DO200.

Los juveniles ( $n=76$ por tanque) tuvieron un peso promedio inicial de $3.77 \pm 1.15 \mathrm{~g}$ (Promedio \pm DE). El periodo de crecimiento fue de 355 días, y fueron alimentados con alimento peletizado (Boza et al. 2008), a una ración de 5\% del peso corporal, la cual fue disminuyendo conforme los peces crecieron. El alimento utilizado (Montes de Oro) varió de $2 \mathrm{~mm}$ al inicio a $4 \mathrm{~mm}$ al final del experimento. El análisis proximal del alimento fue de 50\% de proteína, 17.5\% de carbohidratos y $11.8 \%$ de lípidos, $16 \%$ de ceniza y una humedad del 5\%. Para eliminar los desechos de los peces y restos de alimento, el fondo de los tanques fue sifoneado una vez al día. Los peces fueron pesados de manera individual con una balanza digital Denver Instrument MX612 ( $\pm 0.01 \mathrm{~g}, n=30$ ), al menos dos veces al mes, durante los primeros 3 meses, y luego cada dos meses hasta completar el periodo de 355 días. Una vez pesados, los peces fueron devueltos al respectivo tanque.

Los parámetros biológicos fueron calculados en base a Gunther y Boza (1992), donde el crecimiento fue determinado mediante el coeficiente de crecimiento G según:

$$
\mathrm{G}=\left(\mathrm{P}_{\mathrm{f}}^{(1 / 3)}-\mathrm{P}_{\mathrm{i}}^{(1 / 3)}\right) / \mathrm{t}
$$

Donde $G$ es el coeficiente de crecimiento, $P_{f}=$ Peso final $(g), P_{i}=$ Peso inicial $(g)$, y t es el periodo de días entre los dos pesos.

Con los datos anteriores se calcularon las variables de crecimiento según:

$\mathrm{C}=\mathrm{P}_{\mathrm{f}}-\mathrm{P}_{\mathrm{i}}$

donde $\mathrm{C}=$ crecimiento $(\mathrm{g})$;

$\mathrm{TC}=\mathrm{C} / \mathrm{t}$

donde $\mathrm{TC}=$ Tasa de Crecimiento diario $\left(\mathrm{g} \mathrm{d}^{-1}\right)$;

$\mathrm{TEC}=\left(\left(\ln \mathrm{P}_{\mathrm{f}}-\ln \mathrm{P}_{\mathrm{i}}\right) / \mathrm{t}\right) * 100$

donde $\mathrm{TEC}=$ Tasa específica de crecimiento $\left(\% \mathrm{PC} \mathrm{d}^{-1}\right), \mathrm{PC}=$ Peso corporal, $\ln =$ logaritmo natural; $y$ 
ISSN Electrónico: 2215-3470 DOI: http://dx.doi.org/10.15359/ru.30-2.5
UNICIENCIA Vol. 30, No. 2, pp. 63-74. Julio-diciembre, 2016.

URL: www.revistas.una.ac.cr/uniciencia Email: revistauniciencia@una.cr

$\mathrm{TCR}=\left(\mathrm{C} /\left(\mathrm{PC}_{\mathrm{g}}\right) / \mathrm{t}\right.$
donde $\mathrm{TCR}=$ Tasa de crecimiento relativo $\left(\mathrm{g} \mathrm{g}^{-1} \mathrm{~d}^{-1}\right), \mathrm{PC}_{\mathrm{g}}=\operatorname{EXP}^{((\ln \mathrm{Pf}+\ln \mathrm{Pi}) / 2)}$

$\mathrm{FCA}=\mathrm{C} /$ Alimento consumido

donde FCA = Factor de conversión alimenticia

Las variables de Crecimiento (C), Tasa de Crecimiento (TC), la Tasa Específica de Crecimiento (TEC), y la Tasa de Crecimiento Relativa (TCR) fueron graficadas en función del tiempo, y se calculó el valor de regresión $\left(\mathrm{R}^{2}\right)$, y la curva de tendencia de los datos en el tiempo.

\section{Resultados}

Durante todo el periodo de experimentación en los tanques, la corvina aguada mostró un comportamiento tranquilo, pasando la mayoría del tiempo en la parte sombreada del tanque. Los peces aceptaron la dieta peletizada, fueron fáciles de manipular durante la pesada y no se dio mortalidad durante el periodo de investigación.

El promedio de oxígeno disuelto fue de $7.12 \pm 1.13 \mathrm{mg} \mathrm{L}^{-1}$, con un mínimo de $5.68 \mathrm{mg} \mathrm{L}^{-1}$, y un máximo de $9.13 \mathrm{mg} \mathrm{L}^{-1}$. El promedio de salinidad registrado fue de $31.92 \pm 1.65$ ups, con un mínimo de 29.84 ups y un máximo de 34.71 ups. La temperatura promedio fue de $27.54 \pm 0.63^{\circ} \mathrm{C}$, con un mínimo de $26.50^{\circ} \mathrm{C}$, y un máximo de $28.60^{\circ} \mathrm{C}$.

En la tabla 1 se muestran los resultados correspondientes a los parámetros de crecimiento calculados durante el periodo de estudio. Se observó un incremento en el peso de los juveniles a través del tiempo con un alto coeficiente de correlación $\left(\mathrm{R}^{2}=0.90\right)$, con un peso promedio inicial

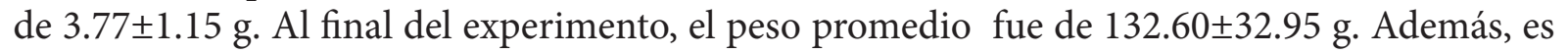
apreciable una mayor varianza en la distribución de tallas por pesos, la cual se refleja en las desviaciones estándar para cada mes (figura 1).

El incremento en peso de los juveniles a través del tiempo mostró una variación en la distribución de tallas, con individuos de pesos superiores y otro grupo con pesos inferiores (figura 2). Al inicio el peso mínimo fue de $1.0 \mathrm{~g}$, y el máximo de $7.0 \mathrm{~g}$, mientras que al final del experimento el mínimo fue de 78.5 g y el máximo de $219.3 \mathrm{~g}$.

El crecimiento (C) por periodos se observa en la tabla 1, el cual tuvo un incremento similar a través del tiempo con un alto coeficiente de correlación $\left(\mathrm{R}^{2}=0.94\right)$. El valor inicial en el crecimiento promedio fue de $5.30 \mathrm{~g}$ hasta $34.6 \mathrm{~g}$ al final del periodo de 355 días (figura 3 ), con un total para todo el periodo de $117.3 \mathrm{~g}$. 
UNICIENCIA Vol. 30, No. 2, pp. 63-74. Julio-diciembre, 2016.

URL: www.revistas.una.ac.cr/uniciencia

Email: revistauniciencia@una.cr
ISSN Electrónico: 2215-3470

DOI: http://dx.doi.org/10.15359/ru.30-2.5

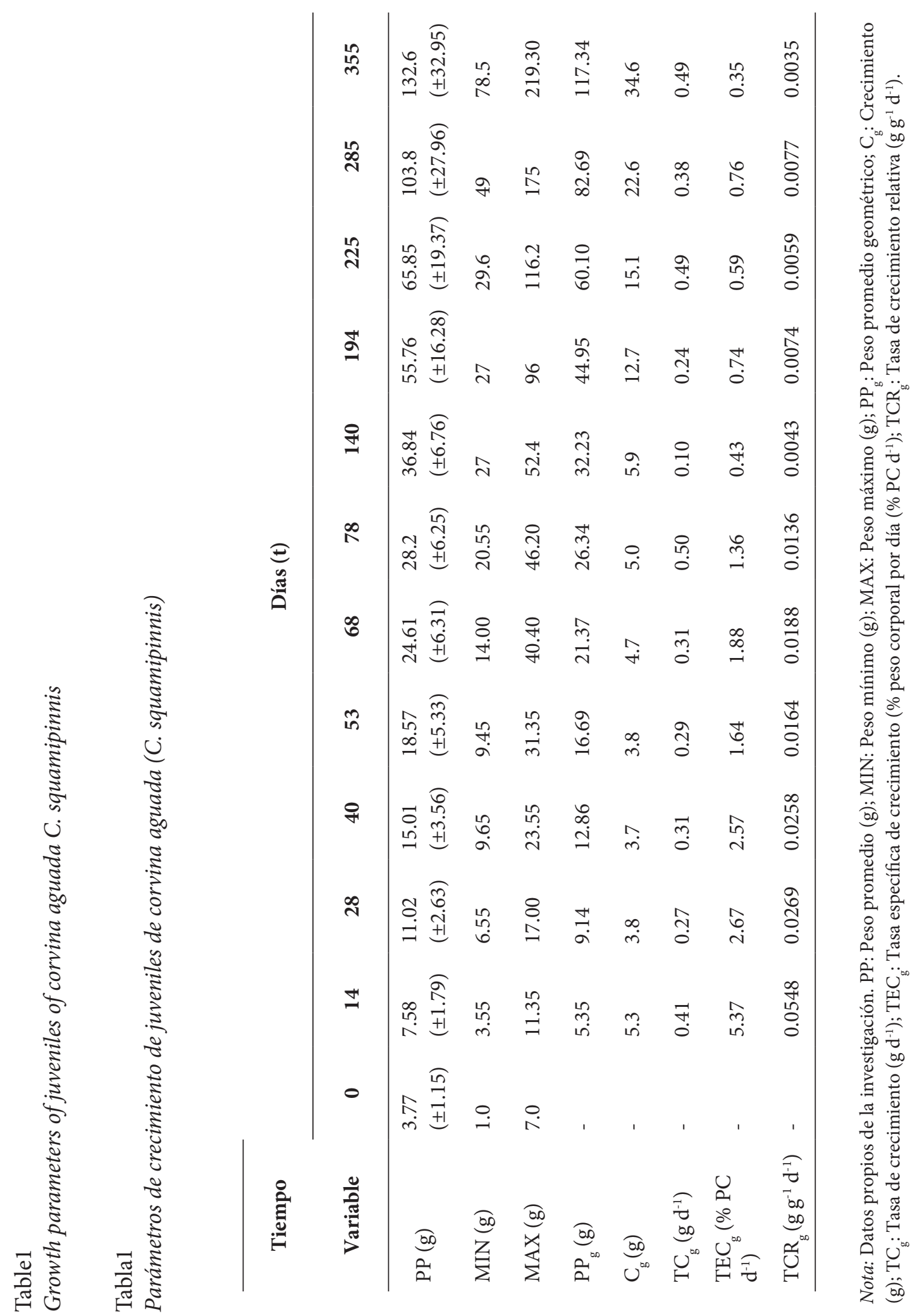


ISSN Electrónico: 2215-3470 DOI: http://dx.doi.org/10.15359/ru.30-2.5
UNICIENCIA Vol. 30, No. 2, pp. 63-74. Julio-diciembre, 2016.

URL: www.revistas.una.ac.cr/uniciencia Email: revistauniciencia@una.cr

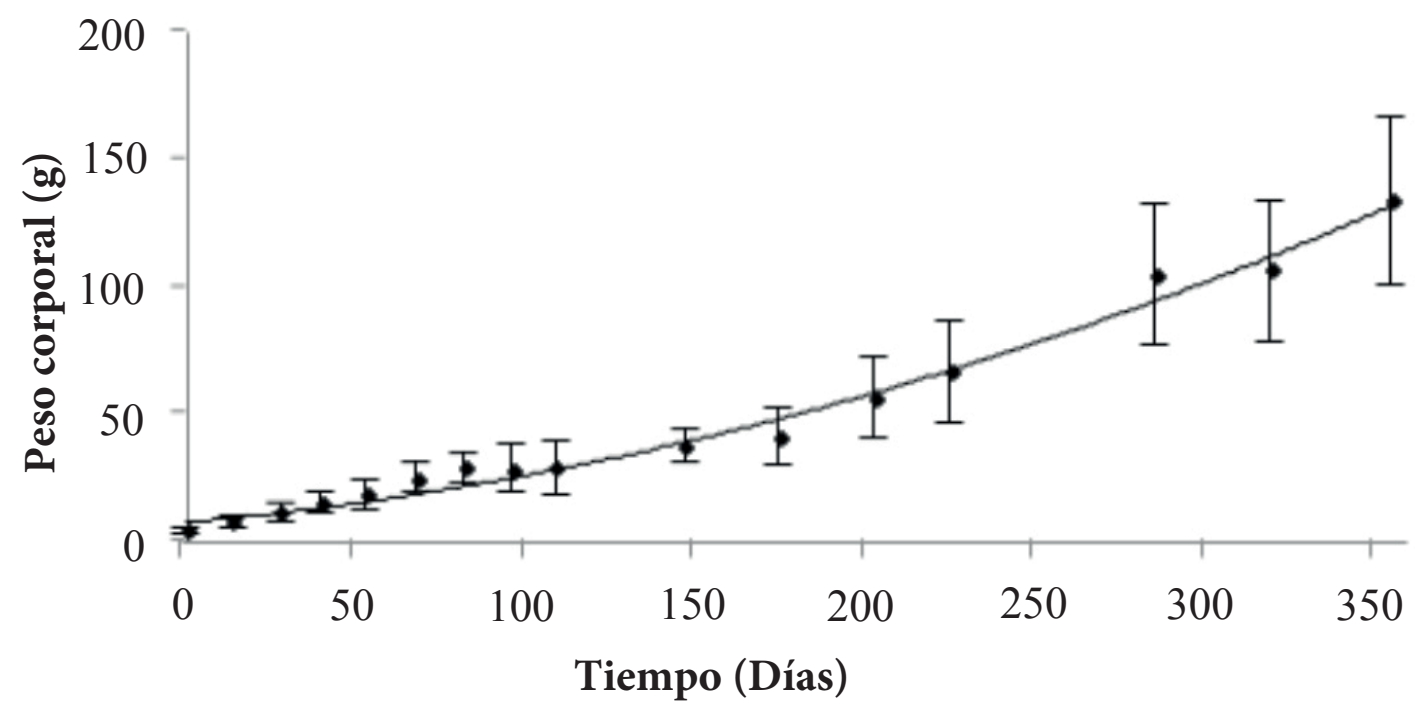

Figura 1. Curva de crecimiento ( $\mathrm{g} \pm \mathrm{DE}$ ) de la corvina aguada (C. squamipinnis) alimentadas con balanceado por un periodo de 355 días. Datos propios de la investigación.

Figure 1. Growth curve ( $\mathrm{g} \pm \mathrm{DE})$ of weakfish (C. squamipinnis) feeding with balanced feed during a period of 355 days

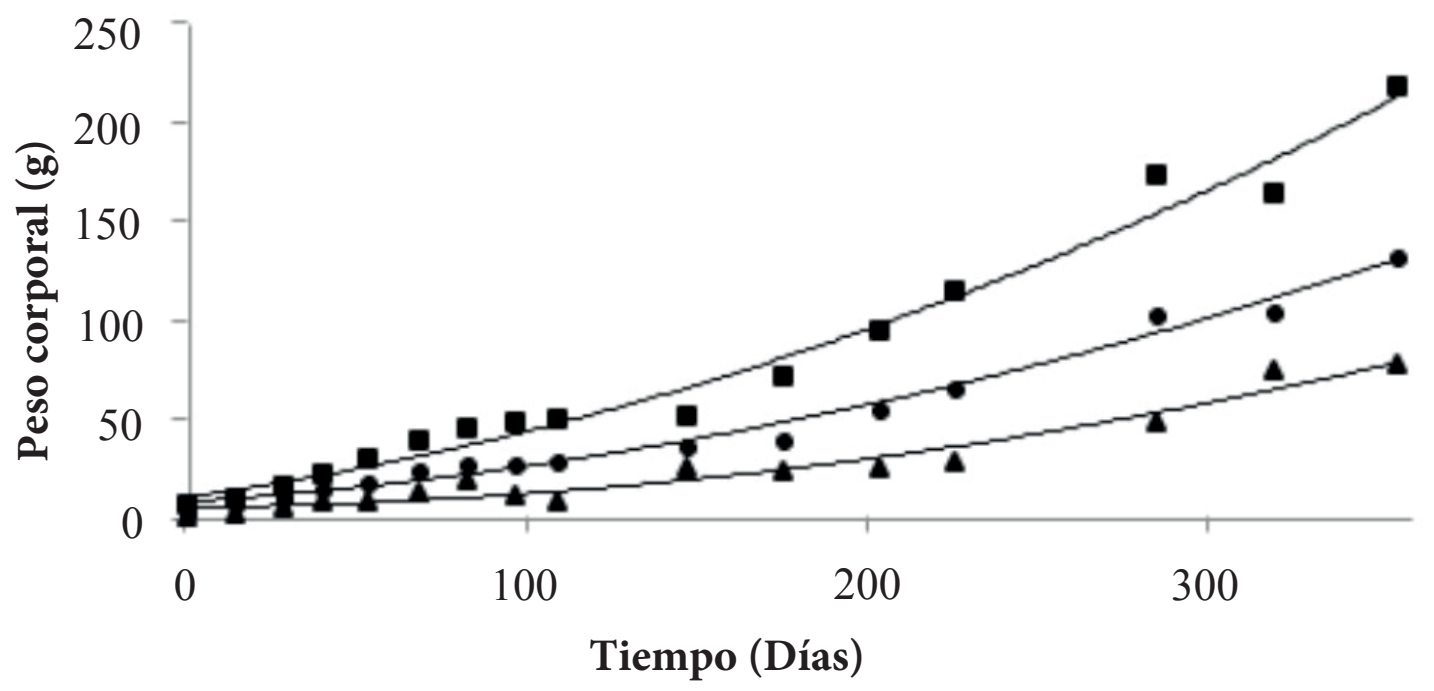

Figura 2. Peso corporal promedio (g, círculos), pesos máximos (g, cuadros), y pesos mínimos (g, triángulos) por pesada, durante el crecimiento de juveniles de corvina aguada (C. squamipinnis). Datos propios de la investigación.

Figure 2. Mean body weight (g, circles), maximum weight (g, squares), minimum weight (g, triangles) during the growth of juveniles of weakfish (C. squamipinnis)

Uniciencia es una revista de acceso abierto/ Uniciencia is an Open Access Journal. 
UNICIENCIA Vol. 30, No. 2, pp. 63-74. Julio-diciembre, 2016.

Email: revistauniciencia@una.cr

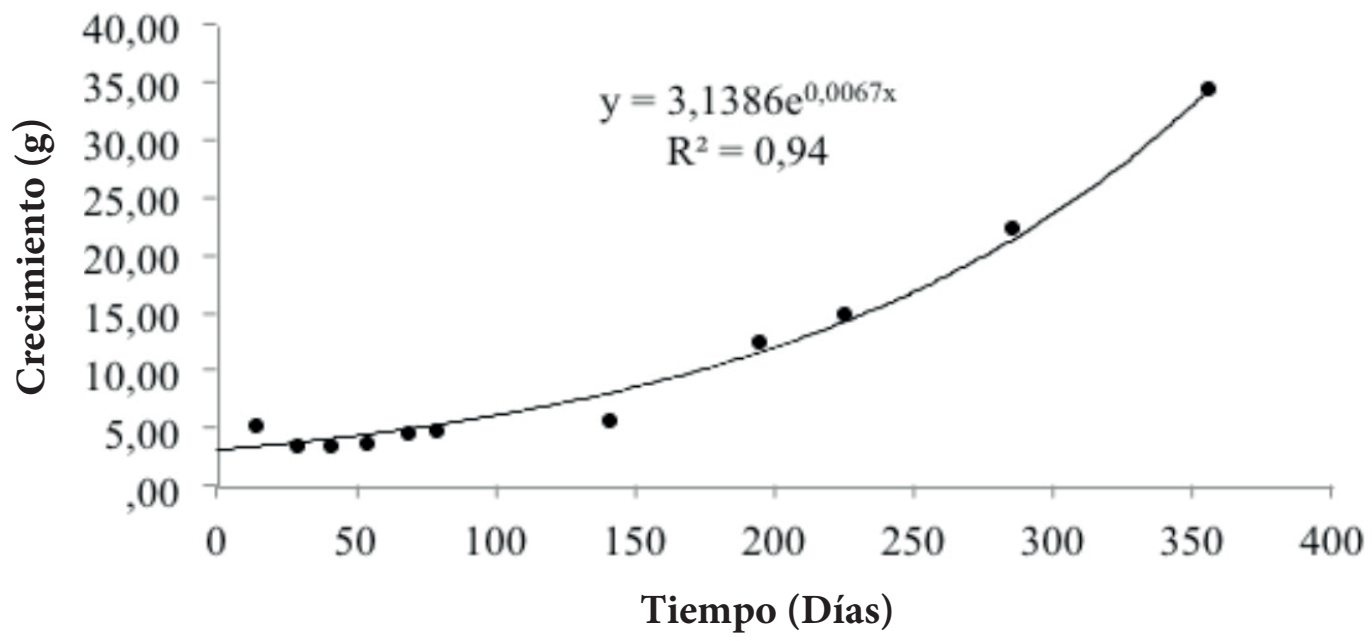

Figura 3. Growth by weight period (g) for juveniles of weakfish (C. squamipinnis).

Figura 3. Crecimiento por pesada (g) para juveniles de corvina aguada (C. squamipinnis). Datos propios de la investigación.

La tasa de crecimiento (TC ) (tabla 1, figura 4) fluctuó de $0.10 \mathrm{~g} \mathrm{~d}^{-1}$ hasta $0.51 \mathrm{~g} \mathrm{~d}^{-1}$ según el periodo de muestreo, mientras que para todo el periodo de engorde alcanzó un valor de $0.36 \mathrm{~g} \mathrm{~d}^{-1}$ a los 355 días $\left(\mathrm{R}^{2}=0.0392\right)$. Contrario a lo observado para el crecimiento y la tasa de crecimiento, la tasa específica de crecimiento $\left(\mathrm{TEC}_{\mathrm{g}}\right.$ ) disminuyó con el tiempo, inició con valores altos (5.37\% $\left.\mathrm{PC} \mathrm{d}^{-1}\right)$, y fue al final del periodo de $0.35 \% \mathrm{PC} \mathrm{d}^{-1}$, mientras que para todo el periodo de engorde fue de $1.00 \% \mathrm{PC} \mathrm{d}^{-1}$ ) (figura 5).

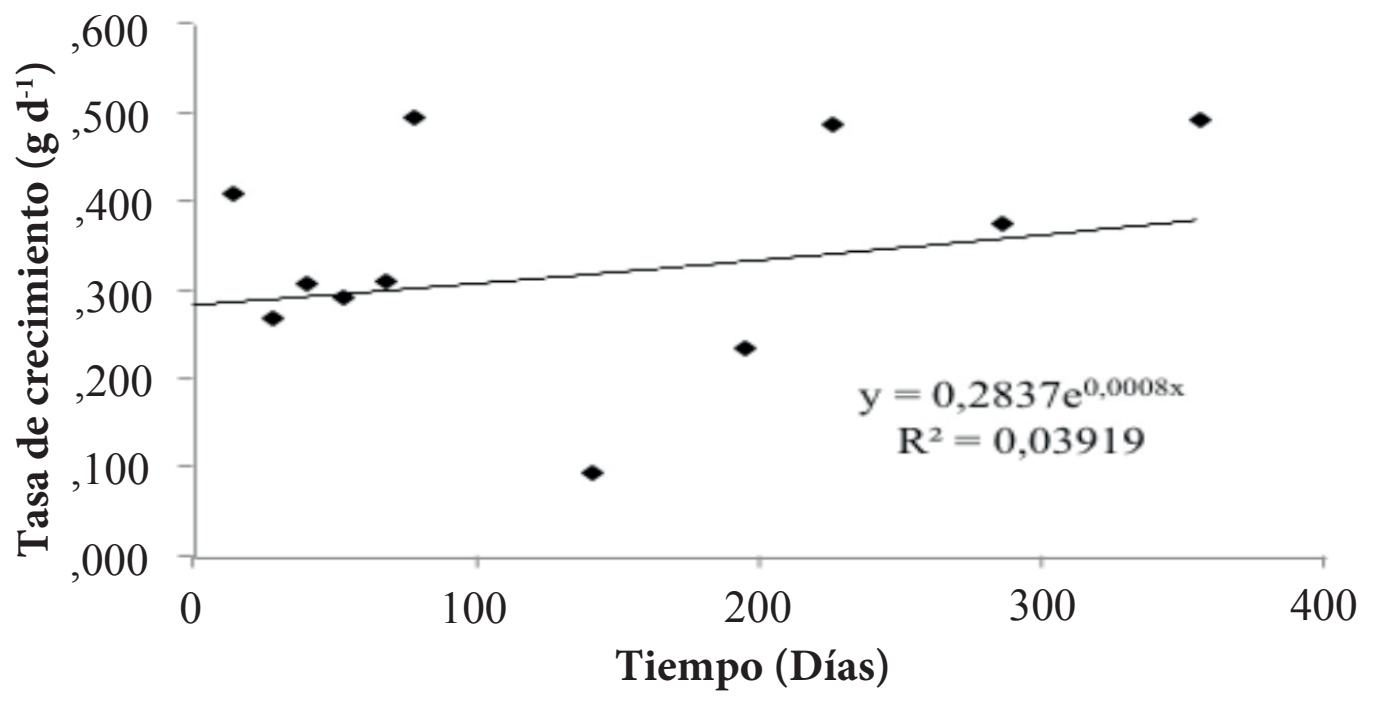

Figura 4. Tasa de crecimiento ( $\mathrm{TC}_{\mathrm{g}}, \mathrm{g} \mathrm{d}^{-1}$ ) de los juveniles de corvina aguada (C. squamipinnis). Datos propios de la investigación.

Figure 4. Growth rate $\left(\mathrm{GR}_{\mathrm{g}}, \mathrm{g} \mathrm{d}^{-1}\right)$ for juveniles of weakfish (C. squamipinnis). 
ISSN Electrónico: 2215-3470 DOI: http://dx.doi.org/10.15359/ru.30-2.5
UNICIENCIA Vol. 30, No. 2, pp. 63-74. Julio-diciembre, 2016.

URL: www.revistas.una.ac.cr/uniciencia Email: revistauniciencia@una.cr

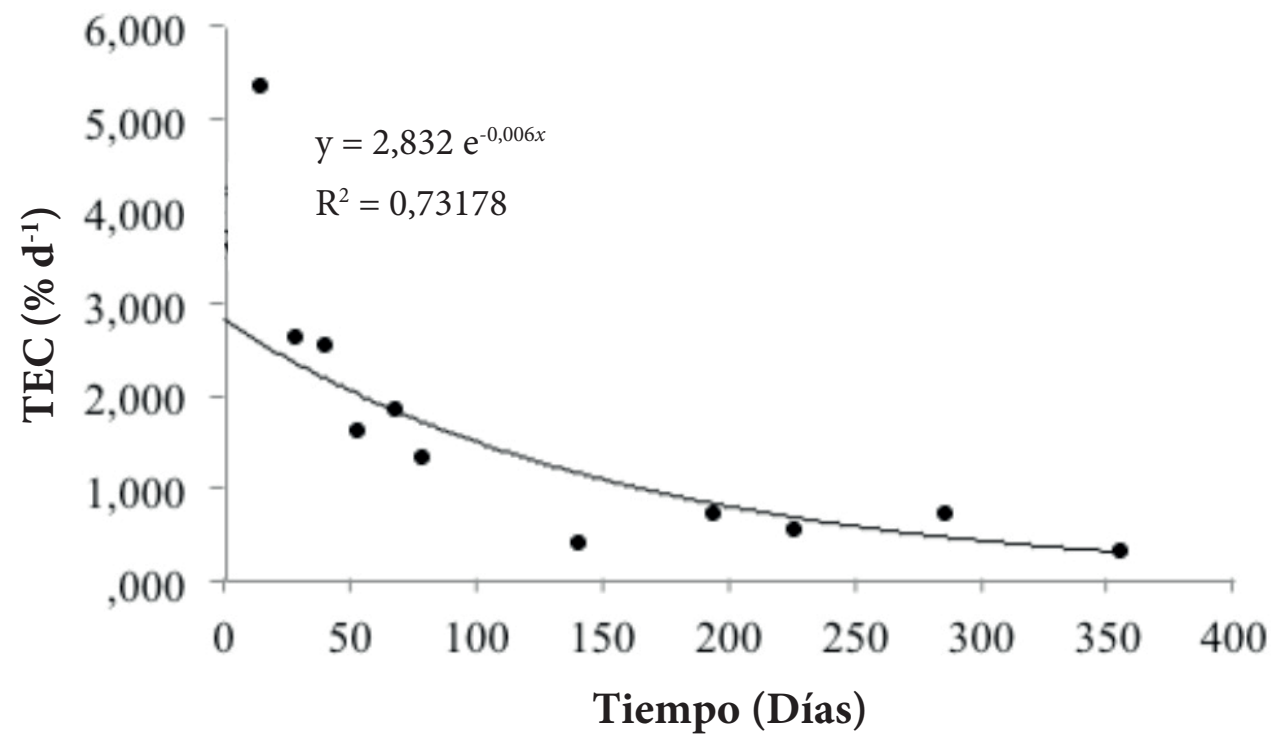

Figura 5. Tasa específica de crecimiento $\left(\mathrm{TEC}_{\mathrm{g}}, \% \mathrm{PC} \mathrm{d}^{-1}\right.$ ) para los juveniles de corvina aguada (C. squamipinnis). Datos propios de la investigación.

Figure 5. Specific growth rate $\left(\mathrm{SGR}_{\mathrm{g}}, \% \mathrm{~d}^{-1}\right)$ for juveniles of weakfish (C. squamipinnis).

De igual manera, la tasa de crecimiento relativo $\left(\mathrm{g} \mathrm{g}^{-1} \mathrm{~d}^{-1}\right)$ fue disminuyendo en el tiempo (figura 6), al inicio fue de $0.0548 \mathrm{~g} \mathrm{~g}^{-1} \mathrm{~d}^{-1}, \mathrm{y}$ llegó al final del periodo de experimentación a 0.0035 $\mathrm{g} \mathrm{g}^{-1} \mathrm{~d}^{-1}$. Para todo el periodo la TCR $\mathrm{g}$ tuvo un valor de $0.016 \mathrm{~g} \mathrm{~g}^{-1} \mathrm{~d}^{-1}$.

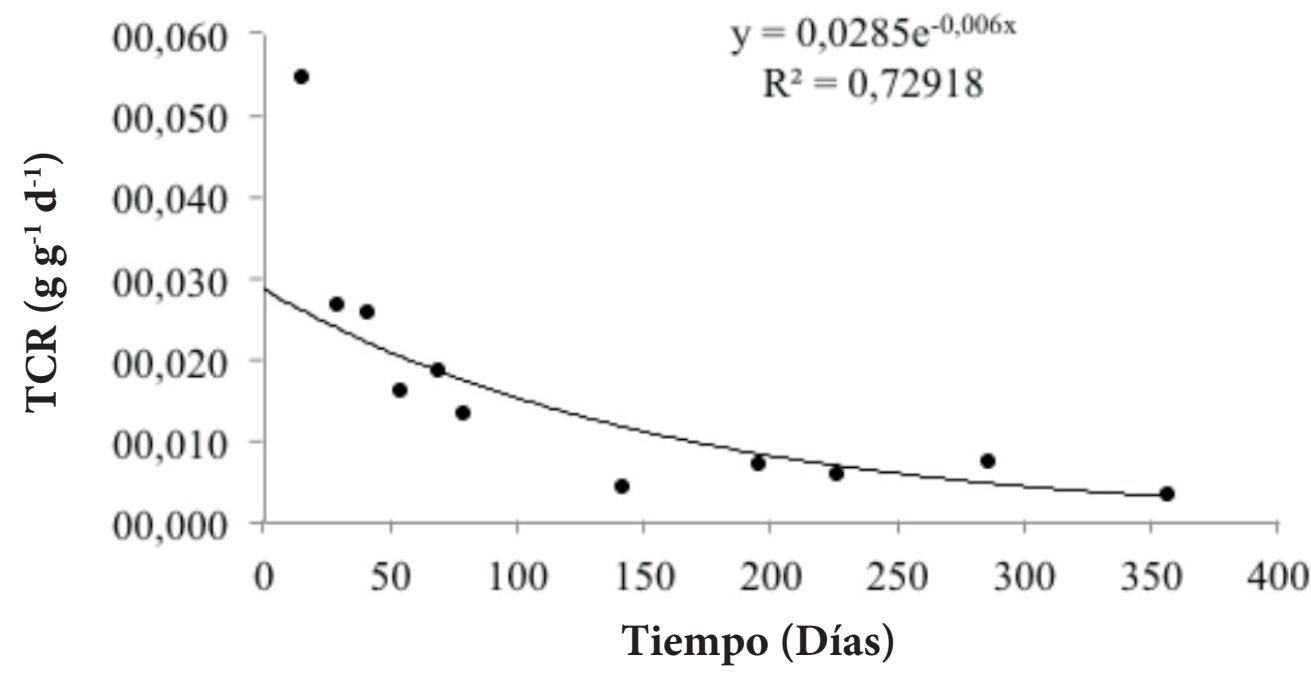

Figura 6. Tasa de crecimiento relativo ( $\mathrm{TCR}_{\mathrm{g}} \mathrm{g} \mathrm{g}^{-1} \mathrm{~d}^{-1}$ ) para juveniles de corvina aguada (C. squamipinnis). Datos propios de la investigación.

Figure 6. Relative growth rate ( $\left.\mathrm{RGR}_{\mathrm{g}} \mathrm{g} \mathrm{g}^{-1} \mathrm{~d}^{-1}\right)$ for juveniles of weakfish (C. squamipinnis).

El factor de conversión alimenticia fue de $1.8 \pm 0.4$ durante todo el periodo de estudio, $y$ varió de 1.5 a 2.7 en dependencia del periodo de alimentación. 
UNICIENCIA Vol. 30, No. 2, pp. 63-74. Julio-diciembre, 2016.

ISSN Electrónico: 2215-3470

URL: www.revistas.una.ac.cr/uniciencia

DOI: http://dx.doi.org/10.15359/ru.30-2.5

Email: revistauniciencia@una.cr

\section{Discusión}

El oxígeno disuelto, la salinidad y la temperatura presentaron las condiciones similares a las del Golfo de Nicoya. La estabilidad en las condiciones ambientales es característica de los países tropicales como Costa Rica, con fluctuaciones muy pequeñas a lo largo del año. Esto favorece el crecimiento de los peces, en especial de las corvinas, cuyos parámetros de crecimiento pueden ser afectados principalmente por la temperatura y la salinidad. Esto ha sido comprobado para A. japonicus en Sur África, al colocar juveniles criados en cautiverio a diferentes salinidades, los parámetros de crecimiento se vieron disminuidos a bajas salinidades (5 ups y 12 ups), y mejoraron a altas salinidades (35 ups) (Bernetzeder et al., 2010). En este estudio, la salinidad se mantuvo entre los 30 - 35 ups, valores que caracterizan las salinidades a lo largo del periodo de experimentación en el Golfo de Nicoya.

El crecimiento de los juveniles de la corvina aguada mostró un aumento exponencial del crecimiento y un incremento en la varianza que se reflejó, a su vez, en el aumento de las desviaciones. Dichas desviaciones se expresan en los valores máximos y mínimos observados durante los muestreos realizados a lo largo del periodo de experimentación. La heterogeneidad poblacional sigue un comportamiento similar al reportado en la bibliografía. En general, para los peces es normal que la población se vuelva más heterogénea a medida que pasan los días, y también es influenciada por factores como la talla inicial, diferencias en las tasas de crecimiento individuales y la mortalidad específica por grupos de tallas (Huston y DeAngelis, 1987).

Dentro de los factores biológicos que determinan el potencial económico de una especie para su producción en acuicultura, se encuentra el crecimiento (Collet, 2007). El crecimiento observado para la especie en estudio a lo largo del experimento y la fuerte correlación observada entre el tiempo y la tasa de crecimiento parecen indicar que la corvina aguada (C. squamipinnis) podría ser una especie con potencial para su producción con fines acuícolas.

En la bibliografía se ha comparado el crecimiento de otras especies de corvina como la corvina mediterránea (A. regius), la cual durante el preengorde ha demostrado tener una tasa específica de crecimiento (TEC) por día superior a la de otras especies como la dorada (Sparus aurata), el bocinegro (Pagrus pagrus), el sargo (Diplodus sargus sargus) y la hurta (Pagrus auriga) (Cárdenas, 2011).

Cárdenas (2011) comparó el preengorde de algunas especies de corvinas. En A. japonicus el peso se incrementó de $7.2 \mathrm{~g}$ a $41.9 \mathrm{~g}(56 \mathrm{~d})$ con una TEC de 3.25\% $\mathrm{PC} \mathrm{d}^{-1}$, en $S$. ocellatus el peso se incrementó de $3.8 \mathrm{~g}$ a $49.2 \mathrm{~g}(80 \mathrm{~d})$ con una TEC de $3.32 \% \mathrm{PC} \mathrm{d}^{-1}$, mientras que en $U$. cirrosa el peso se incrementó de $23 \mathrm{~g}$ a $68 \mathrm{~g}$ en 84 días, con una TEC de $0.51 \% \mathrm{PC} \mathrm{d}^{-1}$. En el caso específico de la corvina mediterránea, el autor reportó TEC que van desde 1.7 - 2.8\% $\mathrm{PC} \mathrm{d}^{-1}(17.5 \mathrm{~g}-18.4 \mathrm{~g}$ al inicio, hasta $27.3 \mathrm{~g}-36.6 \mathrm{~g}$ al final). En la corvina blanca, Atractoscion nobilis, alimentada con piensos con una proteína de 49\%, se obtuvieron TC de $0.39 \mathrm{~g} \mathrm{~d}^{-1}$ (López y Durazo, 2008). Para este estudio, los juveniles de corvina aguada pasaron de $3.77 \mathrm{~g}$ a $24.61 \mathrm{~g}$ en 68 días, con una TC de $0.31 \mathrm{~g} \mathrm{~d}^{-1}$, y una TEC de $1.88 \% \mathrm{PC} \mathrm{d}^{-1}$. Esta diferencia entre algunas de las especies estudiadas y la corvina aguada probablemente se debió a las diferencias en factores ambientales, condiciones de cultivo, $y$ al factor genético intrínseco de la especie.

En este estudio, las corvinas aguadas incrementaron su peso de $3.77 \pm 1.15 \mathrm{~g}$ a $132.60 \pm 32.96$ $\mathrm{g}$ en un periodo de 356 días, obteniendo un TEC de $1.00 \% \mathrm{PC} \mathrm{d}^{-1}$. Cárdenas (2011) resume los estudios realizados en diferentes condiciones y especies. Para A. japonicus, dos estudios iniciaron con pesos de $1 \mathrm{~g}$, y llegaron a pesos de $800 \mathrm{~g}$ (480 días) y $1200 \mathrm{~g}$ (720 días), obtienen una TEC de $0.98 \% \mathrm{PC} \mathrm{d}^{-1}$ y de $1.39 \% \mathrm{PC} \mathrm{d}^{-1}$, respectivamente. C. gilberti engordada en viveros flotantes incrementó su peso de $120 \mathrm{~g}$ a 600 g, en dos ensayos con una duración de 237 días y 396 días, y 
ISSN Electrónico: 2215-3470 DOI: http://dx.doi.org/10.15359/ru.30-2.5
UNICIENCIA Vol. 30, No. 2, pp. 63-74. Julio-diciembre, 2016. URL: www.revistas.una.ac.cr/uniciencia Email: revistauniciencia@una.cr

obtuvo una TEC de $0.68 \% \mathrm{PC} \mathrm{d}^{-1}$ y de $0.40 \% \mathrm{PC} \mathrm{d}^{-1}$, respectivamente. S. ocellatus en estanques, creció de 2.2 g a 1180 g en 540 días, con un TEC de $1.16 \% \mathrm{PC} \mathrm{d}^{-1}$, y en otro ensayo en viveros flotantes de $2 \mathrm{~g}$ a $800 \mathrm{~g}$ en $360 \mathrm{~d}$, con una TEC de $1.66 \% \mathrm{PC} \mathrm{d}^{-1}$. Varios estudios fueron realizados por diferentes autores en condiciones diferentes, iniciando con pesos de $13 \mathrm{~g}-89 \mathrm{~g}$, y finalizando en $822 \mathrm{~g}-1099 \mathrm{~g}$, en periodos de 12 - 24 meses, con TEC de $0.46 \% \mathrm{PC} \mathrm{d}^{-1}-1.04 \% \mathrm{PC} \mathrm{d}^{-1}$.

El valor encontrado en este estudio es inferior a los reportados en la bibliografía, sin embargo, se deben realizar más ensayos con diferentes dietas y exclusivas que satisfagan las necesidades nutricionales de las corvinas. En otras especies de esciánidos como el meagre café, S. umbra, se ha reportado un crecimiento de $5.3 \mathrm{~g}$ a $250 \mathrm{~g}$ en 29 meses de cultivo, considerado un crecimiento bajo con respecto al obtenido por Sparus auratus y Dicentrarchus labrax, especies que llegan a los $400 \mathrm{~g}$ en ese mismo periodo (Chatzifotis et al., 2006).

El FCA obtenido en este estudio se encuentra en el rango reportado por Cárdenas (2011) para especies de esciánidos. Para $A$. japonicus criado en estanques y tanques se obtuvieron valores de 1.1, para C. gilberti criada en viveros flotantes se obtuvieron valores de 2.0, y para S. ocellatus criados en estanques el valor fue de 2.4, mientras que en viveros flotantes el FCA estuvo en el rango de 1.6 a 1.8.

Tomando en cuenta los resultados de crecimiento observados en este estudio preliminar para esta especie de corvina autóctona del Pacífico Americano, su comportamiento en cautiverio, el aceptar el alimento peletizado y su fácil manipulación, se debe considerar a la corvina aguada un candidato a ser tomado en cuenta para el desarrollo de la maricultura en el Golfo de Nicoya. Se recomienda continuar con las investigaciones en el campo de la reproducción, nutrición y manipulación para poder generar un mayor conocimiento de la biología y la tecnología del cultivo de esta especie.

\section{Referencias}

Benetti, D. D., Refik, M., Zink, I., Cavalin, F. G., Sardenberg, B., Palmer, K., Denlinger, B. y Bacoat, D. (2007). Aquaculture of Cobia (Rachycentron canadum) in the Americas and the Caribbean. University of Miami, Rosenstiel School of Marine and Atmospheric Science. Unites States.

Bernatzeder, A. K., Cowley, P. D. y Hecht, Th. (2010). Do juveniles of the estuarine-dependent dusky kob, Argyrosomus japonicus, exhibit optimum growth indices at reduced salinities?. Estuarine, Coastal and Shelf Science, 90, 111-115.

Boza-Abarca, J., Calvo-Vargas, E., Solís-Ortiz, N. y Komen, J. (2008). Desove inducido y crecimiento larval del pargo manchado, Lutjanus guttatus, en la Estación de Biología Marina de Puntarenas, Costa Rica. Ciencias Marinas, 34(2), 239-252.

Boza-Abarca, J., Valverde-Chavarría, S., Calvo-Vargas, E., Ramírez-Alvarado, M. y Rodríguez-Gómez, E. (2011). Inducción hormonal con suspensión de pituitaria de carpa y gonadotropina coriónica humana en el pargo manchado Lutjanus guttatus silvestre y criado en cautiverio. Ciencias Marinas, 37(2), 125-139.

Cárdenas, S. (2011). Cultivo de corvina (Argyrosomus regius). Fundación Observatorio Español de Acuicultura, Madrid, España.

Cárdenas, S. (2012). Biología y acuicultura de corvinas en el mundo. AquaTIC, 37, 1-13.

Chacón, A., Araya, H., Vásquez, A., Brenes, R., Marín, B., Palacios, J., Soto, R., Mejía-Arana, F., Shimazu, Y. y Hiramatsu, K. (2007). Estadísticas pesqueras del Golfo de Nicoya, Costa Rica, 1994-2005. Proyecto Manejo Sostenible de la Pesquería para el Golfo de Nicoya. JICA-UNA-INCOPESCA. Costa Rica.

Chatzifotis, S., Martin-Prat, A.V., Limberis, N., Papandroulakis, N. y Divanach, P. (2006). First data on growth of cultured brown meagre, Sciaena umbra, using diets with different protein and fat contents. Fisheries Science, 72, 83-88. 
UNICIENCIA Vol. 30, No. 2, pp. 63-74. Julio-diciembre, 2016.

Chatzifotis, S., Panagiotidou, M., Papaioannou, N., Pavlidis, M., Nengas, I. y Mylonas, C. C. (2010). Effect of dietary lipid levels on growth, feed utilization, body composition and serum metabolites of meagre (Argyrosomus regius) juveniles. Aquaculture, 307, 65-70.

Collet, P. (2007). Toward the development of a rearing protocol for juvenile dusky kob, Argyrosomus japonicus (Pisces: Sciaenidae) (Tesis de posgrado). Rhodes University, Grahamstown, South Africa.

Connaughton, M. A. y Taylor, M. (1996). Drumming, courtship, and spawning behavior in captive weakfish, Cynoscion regalis. Copeia, (1), 195-199.

Duncan, N., Estévez, A., Porta, J., Carazo, I., Norambuena, F., Aguilera, C., Gairin, I. y Bucci, F. (2012). Reproductive development, GnRHa induced spawning and egg quality of wild meagre (Argyrosomus regius) acclimatized to captivity. Fish. Physiol. Biochem., 38, 1273-1286.

Gunther, J. y Boza, J. (1992). Growth perfomance of Colossoma macropomum (Cuvier) juveniles at different feed rations. Aquaculture and Fisheries Management, 23, 81-93.

Huston, A. y DeAngelis, D. (1987). Size bimodality in monoespecific populations: A critical review of potential mechanisms. The American Naturalist, 129 (5), 678-707.

Jiménez, M. T., Pastor, E., Grau, A., Alconchel, J. I., Sánches, R. y Cárdenas, S. (2005). Revisión del cultivo de esciénidos en el mundo, con especial atención a la corvina Argyrosomus regius (Asso, 1801). Boletín. Instituto Español de Oceanografía, 21(1-4), 169-175.

Lagárdere, P. J. y Marianis, A. (2006). Spawning sounds in meagre Argyrosomus regius recorded in the Gironde estuary, France. Journal of Fish Biology, 69(6), 1697-1708.

López, L. M., Durazo, E., Rodríguez, A., True, C. D. y Viana, M. T. (2006). Composición proximal y perfil de ácidos grasos de juveniles silvestres y cultivados de Totoaba macdonaldi. Ciencias Marinas, 32, 303-309.

López, L. M. y Durazo, E. (2008). Guía para la nutrición y cultivo de corvina blanca. Grupo de nutrición, Universidad autónoma de Baja California, Facultad de Ciencias Marinas, Ensenada, B.C., México.

Marín, B. A. y Vásquez, A. R. A. (2012). Estimación de la talla de primera madurez sexual criterio L50\% (TPM) de la corvina reina Cynoscion albus (Perciforme: Scianidae) bajo condiciones de sobreexplotación de su población en el Golfo de Nicoya, Costa Rica. Documento Técnico $\mathrm{N}^{\circ} 11$, INCOPESCA, Puntarenas, Costa Rica.

Monfort, M. C. (2010). Studies and Reviews $\mathrm{N}^{\circ}$ 89: Present market situation and prospects of meagre (Argyrosomus regius), as an emerging species in Mediterranean Aquaculture. Food and Agriculture Organization of the United Nations (FAO), Roma, Italia.

Mylonas, C. C., Mitrizakis, N., Papadaki, M. y Sigelaki, I. (2013a). Reproduction of hatchery-produced meagre Argyrosomus regius in captivity I. Description of the annual reproductive cycle. Aquaculture, 414-415, 309-317.

Mylonas, C. C., Mitrizakis, N., Castaldo, C. A., Cerviño, Cl. P., Papadaki, M. y Sigelaki, I. (2013b). Reproduction of hatchery-produced meagre Argyrosomus regius in captivity II. Hormonal induction of spawning and monitoring of spawning kinetics, egg production and egg quality. Aquaculture, 414-415, 318-327.

Piccolo, G., Bovera, F., De Riu, N., Marono, S., Salati, F., Cappuccinelli, R. y Moniello, G. (2008). Effect of two different protein/fat ratios of the diet on meagre (Argyrosomus regius) traits. Ital. J. Anim. Sci., 7, 363-371.

Vásquez, A. R. A. y Marín, B. A. (2012). Estudio línea base pesquero del área de pesca de la comunidad de Tárcoles en el Año 2005. Documento Técnico Nº 10, INCOPESCA, Puntarenas, Costa Rica.

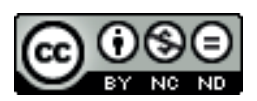

Crecimiento de juveniles de la corvina aguada, Cynoscion squamipinnis criados en cautiverio (Jorge Boza-Abarca y otros) por Revista Uniciencia se encuentra bajo una Licencia CreativeCommons Atribución-NoComercial-SinDerivadas 3.0 Unported. 